October 13, 2018

LBNL-41142, UCB-PTH-98/01

hep-th/9802079

\title{
Conformal Field Theories: From Old to New ${ }^{1}$
}

\author{
J. de Boer and M. B. Halpern \\ Department of Physics, University of California at Berkeley \\ 366 Le Conte Hall, Berkeley, CA 94720-7300, U.S.A. \\ and \\ Theoretical Physics Group, Mail Stop 50A-5101 \\ Ernest Orlando Lawrence Berkeley National Laboratory \\ Berkeley, CA 94720, U.S.A.
}

\begin{abstract}
In a short review of recent work, we discuss the general problem of constructing the actions of new conformal field theories from old conformal field theories. Such a construction follows when the old conformal field theory admits new conformal stress tensors in its chiral algebra, and it turns out that the new conformal field theory is generically a new spin-two gauge theory. As an example we discuss the new spin-two gauged sigma models which arise in this fashion from the general conformal non-linear sigma model.
\end{abstract}

\footnotetext{
${ }^{1}$ To appear in a memorial issue of Theoretical and Mathematical Physics in memory of F.A. Lunev.
} 


\section{Introduction}

We are saddened by the death of Dr. F.A. Lunev and offer this contribution in his honor.

The problem of constructing new conformal field theories from old conformal field theories dates back to $K$-conjugation covariance [1-5], the coset constructions [1-3] and the general affine-Virasoro construction [5-7]. Such a construction follows when the old conformal field theory admits new conformal stress tensors in its chiral algebra. The simplest examples of this construction are the coset constructions, whose (new) spinone gauged WZW actions [8-11] are obtained from the (old) WZW actions. The coset constructions are however a special case of higher symmetry, and the problem of finding the actions of the generic affine-Virasoro constructions was solved in [12-14], where the (new) spin-two gauged WZW actions of these theories were obtained from the (old) WZW action.

Recently, we have extended [15-17] this program to the new conformal field theories which can be obtained in this way from the (old) general non-linear sigma model, and this memorial issue provides an opportunity to review the program here in general terms. The discussion of the second and third sections is based on material originally discussed in $[12,18]$, and the discussion of Section 4 is based on material originally discussed in $[15-17]$.

\section{Action Formulation of a CFT}

Consider a conformal field theory $(\mathrm{CFT}) C_{*}$ with chiral/antichiral stress tensors $T_{*}$, $\bar{T}_{*}$ and associated chiral/antichiral algebras $\mathcal{A}_{*}, \overline{\mathcal{A}}_{*}$

$$
T_{*} \in \mathcal{A}_{*}, \quad \bar{T}_{*} \in \overline{\mathcal{A}}_{*}
$$

where $\mathcal{A}_{*}, \overline{\mathcal{A}}_{*}$ are defined to include all mutually local holomorphic/antiholomorphic objects in the theory. The naive Hamiltonian of $C_{*}$ is

$$
H_{* 0}=\int_{0}^{2 \pi} d \sigma \mathcal{H}_{* 0}, \quad \mathcal{H}_{* 0}=T_{*}+\bar{T}_{*}
$$

but $C_{*}$ is a gauge theory if the centralizers $\mathcal{A}_{*}^{\prime}, \overline{\mathcal{A}}_{*}^{\prime}$ of $\mathcal{H}_{* 0}$ in $\mathcal{A}_{*}, \overline{\mathcal{A}}_{*}$,

$$
\mathcal{A}_{*}^{\prime}=\left\{X(z) \in \mathcal{A}_{*} \mid\left[X(z), T_{*}\right]=0\right\}, \quad \overline{\mathcal{A}}_{*}^{\prime}=\left\{X(\bar{z}) \in \overline{\mathcal{A}}_{*} \mid\left[X(\bar{z}), \bar{T}_{*}\right]=0\right\}
$$


are non-trivial ${ }^{1}$. We assume for simplicity that all elements of the centralizer can be expressed as differential polynomials in terms of a finite local set of basis elements, which we also denote by $\mathcal{A}_{*}^{\prime}, \overline{\mathcal{A}}_{*}^{\prime}$. The centralizers are in fact infinite dimensional $\mathbf{Z}$-graded algebras, and the corresponding positive frequency modes of the centralizers will be denoted by $\mathcal{A}_{*}^{\prime}(+), \overline{\mathcal{A}}_{*}^{\prime}(+)$. Following Gupta and Bleuler, the theory $C_{*}$ can then be described by the Hamiltonian $H_{* 0}$ acting on a physical Hilbert space defined by

$$
\left.\left.\mathcal{A}_{*}^{\prime}(+) \mid \text { phys }\right\rangle=\overline{\mathcal{A}}_{*}^{\prime}(+) \mid \text { phys }\right\rangle=0 .
$$

This means that the physical states are primary under the algebras $\mathcal{A}_{*}^{\prime}, \overline{\mathcal{A}}_{*}^{\prime}$.

When $C_{*}$ has a smooth classical limit, we expect that the theory has an action description, To find the action one must first find the classical limit or Poisson bracket description of the objects and algebras described above. We assume for simplicity that the classical limit of the centralizer contains no central terms, that is, the basis elements of the centralizer form a set of first-class constraints in the language of Dirac. Then the full classical Hamiltonian of the the $\mathrm{CFT} C_{*}$ can be written as

$$
H_{*}=\int d \sigma \mathcal{H}_{*}, \quad \mathcal{H}_{*}=\mathcal{H}_{* 0}+v \cdot \mathcal{A}_{*}^{\prime}+\bar{v} \cdot \overline{\mathcal{A}}_{*}^{\prime}
$$

where $v, \bar{v}$ are Lagrange multipliers, and the action $S_{*}$ of $C_{*}$ is obtained by the usual canonical method. The multipliers $v, \bar{v}$ form world-sheet gauge fields whose spins are those of the corresponding elements of the centralizers $\mathcal{A}_{*}^{\prime}, \overline{\mathcal{A}}_{*}^{\prime}$ of $C_{*}$.

\section{New CFT's}

We focus now on a case of particular interest, when the chiral/antichiral algebras $\mathcal{A}_{*}, \overline{\mathcal{A}}_{*}$ of $C_{*}$ contain two new chiral/antichiral spin-two objects $T$ and $\bar{T}$ which satisfy commuting Virasoro algebras. In this case, one expects the existence of two further chiral/antichiral spin-two tensors $\tilde{T}, \tilde{\bar{T}}$, so that all four stess tensors

$$
T, \tilde{T}, \bar{T}, \tilde{\bar{T}} ; \quad T, \tilde{T} \in \mathcal{A}_{*}, \quad \bar{T}, \tilde{\bar{T}} \in \overline{\mathcal{A}}_{*}
$$

are commuting Virasoro operators. The four new stress tensors sum in pairs to the stress tensors $T_{*}, \bar{T}_{*}$ of $C_{*}$,

$$
T_{*}=T+\tilde{T}, \quad \bar{T}_{*}=\bar{T}+\tilde{\bar{T}}
$$

\footnotetext{
${ }^{1}$ Usually one defines the chiral algebra of a conformal field theory such that these centralizers contain only the unit operator, the gauge degrees of freedom having already been modded out. However, we first allow for a more general situation where the conformal field theory is embedded in a larger gauge-covariant system, modding out later by the gauge degrees of freedom.
} 
and this is called $K$-conjugation covariance [1-5]. This phenomenon is most familiar in the coset constructions (where $T_{*}=T_{g}, T=T_{g / h}, \tilde{T}=T_{h}$ ) and is explicit in the general affine-Virasoro construction, but the phenomenon of $K$-conjugation covariance was argued quite generally in [4].

In the presence of $K$-conjugation covariance we see that $C_{*}$ is a tensor product CFT composed of the $K$-conjugate pair of CFTs $C$ and $\tilde{C}$

$$
\begin{gathered}
C_{*}=C \otimes \tilde{C} \\
C: T, \bar{T}, \quad \tilde{C}: \tilde{T}, \tilde{\bar{T}}
\end{gathered}
$$

whose stress tensors are shown in (3.3b). In what follows we focus on the $C$ theory, but the corresponding description of the $\tilde{C}$ theory can be obtained at any stage of the discussion by $K$-conjugation.

The naive Hamiltonian of the $C$ theory is

$$
H_{0}=\int d \sigma \mathcal{H}_{0}, \quad \mathcal{H}_{0}=T+\bar{T}
$$

and we see that $C$ is generically ${ }^{1}$ a spin-two gauge theory because the Virasoro operators $\tilde{T}$ and $\tilde{\bar{T}}$ are in the centralizer $\mathcal{A}^{\prime}, \overline{\mathcal{A}}^{\prime}$ of $\mathcal{H}_{0}$. In the classical limit, the full Hamiltonian of the generic theory $C$ is therefore

$$
H=\int d \sigma \mathcal{H}, \quad \mathcal{H}=T+\bar{T}+v \tilde{T}+\bar{v} \tilde{\bar{T}}
$$

where $v$ and $\bar{v}$ form a spin-two gauge field on the world-sheet.

Generically, the centralizer of $H_{0}$ is nothing but $\tilde{T}$ and $\tilde{\bar{T}}$ so the Hamiltonian (3.5) is the proper description of the generic new CFT $C$. In special cases of higher symmetry however, we must gauge the new theory $C$ by the full centralizer of $\mathcal{H}_{0}$,

$$
\mathcal{H}=T+\bar{T}+v \cdot \mathcal{A}^{\prime}+\bar{v} \cdot \overline{\mathcal{A}}^{\prime}
$$

where $\mathcal{A}^{\prime}, \overline{\mathcal{A}}^{\prime}$ may satisfy Virasoro algebras, $W_{3}$ algebras, etc. Adding to (3.6) a term proportional to $v \bar{v}$, one can also include local spin-one symmetries, which are associated to affine Lie algebras. The actions of the new CFTs then follow by the usual canonical prescription.

\footnotetext{
${ }^{1}$ The case of the $g / h$ coset constructions is a special case of higher symmetry: These are spin-one gauge theories because the centralizer of $T_{g / h}$ is generated by the $h$-currents, with $T_{h}$ a composite operator.
} 


\section{New Spin-Two Gauged Sigma Models}

We have recently studied this program $[16,17]$ starting with the action of the general conformal non-linear sigma model

$$
S_{*}=S_{G}=\int d^{2} \xi \mathcal{L}_{G}, \quad \mathcal{L}_{G}=\left(G_{i j}+B_{i j}\right) \partial_{+} x^{i} \partial_{-} x^{j}
$$

with conformal stress tensors $T_{G}, \bar{T}_{G}$. Taking the sigma model as a background, we looked for all four new chiral/antichiral stress tensors $T, \tilde{T}, \bar{T}, \tilde{\bar{T}}$ in the generic form

$$
T \sim L_{i j} \partial_{+} x^{i} \partial_{+} x^{j}
$$

and found that they exist in $K$-conjugate pairs

$$
T_{*}=T_{G}=T+\tilde{T}, \quad \bar{T}_{*}=\bar{T}_{G}=\bar{T}+\bar{T}_{G}
$$

under certain conditions on the coefficients $L_{i j}$, given below (see $(4.5 \mathrm{a})$ and $(4.5 \mathrm{~b})$ ). Including the dilaton, the quantum extension of (4.2) and (4.3) has been verified at the one-loop level in $[15,16]$.

Following the program outlined above, we found that the generic new conformal field theories $C=T, \bar{T}$ are described by the following set of new spin-two gauged sigma models

$$
\begin{gathered}
S=\int d^{2} \xi \mathcal{L} \\
\mathcal{L}=\mathcal{L}_{G}+\frac{1}{4 \pi \alpha^{\prime}}\left[\alpha \tilde{L}_{i j} B^{i} B^{j}+\bar{\alpha} \tilde{\bar{L}}_{i j} \bar{B}^{i} \bar{B}^{j}\right. \\
\left.-\left(B^{i}-\partial_{+} x^{i}\right) G_{i j}\left(\bar{B}^{j}-\partial_{-} x^{j}\right)\right] \\
\hat{\nabla}_{i}^{+} \tilde{L}_{j}^{k}=\hat{\nabla}_{i}^{-} \tilde{\bar{L}}_{j}^{k}=0 \\
\tilde{L}_{i}{ }^{j}=2 \tilde{L}_{i}^{k} \tilde{L}_{k}{ }^{j}, \quad \tilde{\bar{L}}_{i}^{j}=2 \tilde{\bar{L}}_{i}^{k} \tilde{\bar{L}}_{k}{ }^{j} .
\end{gathered}
$$

Here $\alpha, \bar{\alpha}$ form a spin-two gauge field, closely related to the multipliers $v, \bar{v}$, and $B$, $\bar{B}$ are auxiliary fields. One new $C=T, \bar{T}$ conformal field theory is obtained for each solution of the conditions in (4.5a), (4.5b). The gradients $\hat{\nabla}^{ \pm}$in (4.5a) are generalized covariant derivatives with torsion. See [17] for further details, including the spin-two gauge invariance of these actions and their non-linear form after integrating out the auxiliary fields.

The relations (4.5a), (4.5b) are nothing but the conditions that the classical chiral

algebras are closed, and necessary and sufficient conditions for their solution are also 
discussed in [15-17]. The following explicit examples of this system have been discussed: - the spin-two gauged WZW actions

- $\quad$ the spin-two gauged $g / h$ coset constructions.

In the first case, the action $S_{*}=S_{W Z W}$ is the WZW action and the action $S$ in (4.4a) is the generic affine-Virasoro action [12-14], which describes the generic affine-Virasoro construction. In the second case [17], where $S_{*}=S_{g / h}$ is the sigma model formulation of the coset constructions, the actions $S$ have been identified as the actions of the Lie $h$-invariant CFTs [19], which are those generically irrational CFTs with an extra local $h$ gauge symmetry. (Because they have an extra local $h$ symmetry, the Lie $h$-invariant CFTs are not generic and are therefore not included in the generic spin-two gauged WZW action.)

In the case that the centralizer of $T$ is larger than the Virasoro algebra, we can still construct an action for the $C=T, \bar{T}$ theory. If the centralizer is generated by holomorphic/antiholomorphic polynomials $P_{r}\left(x^{i}, \partial_{+} x^{i}\right), \bar{P}_{r}\left(x^{i}, \partial_{-} x^{i}\right)$, the action is of the form $(4.4 \mathrm{~b})$,

$$
\begin{aligned}
S=\int d^{2} \xi \mathcal{L} & \\
\mathcal{L}=\mathcal{L}_{G}+\frac{1}{4 \pi \alpha^{\prime}} & {\left[\sum_{r} \alpha_{r} P_{r}\left(x^{i}, B^{i}\right)+\sum_{r} \bar{\alpha}_{r} \bar{P}_{r}\left(x^{i}, \bar{B}^{i}\right)\right.} \\
& \left.\quad-\left(B^{i}-\partial_{+} x^{i}\right) G_{i j}\left(\bar{B}^{j}-\partial_{-} x^{j}\right)\right]
\end{aligned}
$$

where $\alpha \tilde{L}_{i j} B^{i} B^{j}$ has been replaced by $\sum_{r} \alpha_{r} P_{r}\left(x^{i}, B^{i}\right)$, and similarly for the term involving $\bar{\alpha}$. It is quite remarkable that by introducing auxiliary fields this large class of actions can be brought to this simple polynomial form. Integrating out the auxiliary fields yields the non-linear form of these actions, which are also non-local in the general case.

The action (4.4b) and its generalizations could serve as a starting point for a perturbative BRST quantization of the new conformal field theories (see [13]). It would be interesting to study this and the relation to non-critical string theories in more detail.

The action (4.4b) describes the new conformal field theories in a conformal gauge for the $C$ theory, that is, the world-sheet metric $h_{m n}$ of the $C$ theory is proportional to $\delta_{m n}$. By also gauging the stress tensors $T, \bar{T}$ of the $C$ theory

$$
H_{2}=\int d \sigma \mathcal{H}_{2}, \quad \mathcal{H}_{2}=u T+\bar{u} \bar{T}+v \tilde{T}+\bar{v} \tilde{\bar{T}}
$$

we obtain a "doubly-gauged" action $[12-14,17]$ for the new CFTs with an arbitrary worldsheet metric $h_{m n}$ for the $C$ theory, which is composed of the gauge fields $u, \bar{u}$. In fact, the theory now contains two world-sheet metrics, where the " $K$-conjugate" metric $\tilde{h}_{m n}$ 
(the world-sheet metric of the $\tilde{C}$ theory) is composed of the old gauge fields $v, \bar{v}$, and the description of the $K$-conjugate pair of conformal field theories $C, \tilde{C}$ is now formally symmetric. To describe the CFT $C$, one views $h_{m n}$ as a fixed world-sheet metric and integrates out the spin-two gauge field $\tilde{h}_{m n}$, and vice-versa to describe the CFT $\tilde{C}$.

The procedure discussed above interprets the $K$-conjugate pair $C, \tilde{C}$ of CFTs as separate conformal field theories. An alternative procedure is to integrate out both spin-two gauge fields $h_{m n}$ and $\tilde{h}_{m n}$, which defines a new class of string theories where the physical states are primary under a $K$-conjugate pair of Virasoro operators. The first example of

this kind of theory was the "spin-orbit" model of [1] (see also [7]) and this new class of string theories may also be related to the models of [20].

\section{Acknowledgements}

This research is supported in part by NSF grant PHY-95-14797 and DOE grant DEAC03-76SF00098. JdB is a fellow of the Miller Institute for Basic Research in Science.

\section{References}

[1] K. Bardakçi and M.B. Halpern, Phys. Rev. D3 (1971) 2493.

[2] M.B. Halpern, Phys. Rev. D4 (1971) 2398.

[3] P. Goddard, A. Kent and D. Olive, Phys. Lett. B152 (1985) 88.

[4] E. Kiritsis, Mod. Phys. Lett. A4 (1989) 437.

[5] M.B. Halpern and E. Kiritsis, Mod. Phys. Lett. A4 (1989) 1373; Erratum ibid. A4 (1989) 1797.

[6] A.Yu Morozov, A.M. Perelomov, A.A. Rosly, M.A. Shifman and A.V. Turbiner, Int. J. Mod. Phys. A5 (1990) 803.

[7] M.B. Halpern, E. Kiritsis, N.A. Obers and K. Clubok, "Irrational Conformal Field Theory", Physics Reports 265 (1996) 1.

[8] K. Bardakçi, E. Rabinovici and B. Säring, Nucl. Phys. B299 (1988) 151; D. Altschuler, K. Bardakçi and E. Rabinovici, Comm. Math. Phys. 118 (1988) 241.

[9] K. Gawedski and A. Kupainen, Phys.Lett. B215 (1988) 119; Nucl. Phys. B320 (1989) 625.

[10] D. Karabali, Q-H. Park, H.J. Schnitzer and Z. Yang, Phys. Lett. B216 (1989) 307.

[11] D. Karabali and H.J. Schnitzer, Nucl. Phys. B329 (1990) 649.

[12] M.B. Halpern and J.P. Yamron, Nucl. Phys. B351 (1991) 333.

[13] J. de Boer, K. Clubok and M.B. Halpern, Int. J. Mod. Phys. A9 (1994) 2451. 
[14] K. Clubok and M.B. Halpern, The generic world-sheet action of irrational conformal field theory, in: "Strings'95", I. Bars et al., eds., World Scientific, Singapore (1996).

[15] J. de Boer and M.B. Halpern, Int. J. Mod. Phys. A12 (1997) 1551.

[16] J. de Boer and M.B. Halpern, "Unification of the General Non-Linear Sigma Model and the Virasoro Master Equation", to appear in the Proceedings of the NATO Workshop "New Developments in Quantum Field Theory", Zakopane, June 1997, Plenum Press, N.Y.

[17] J. de Boer and M.B. Halpern, "New Spin-Two Gauged Sigma Models and General Conformal Field Theory", to appear.

[18] M.B. Halpern, Recent progress in irrational conformal field theory, in: "Strings 1993", M.B. Halpern et al., eds., World Scientific, Singapore (1995).

[19] M.B. Halpern, E. Kiritsis and N.A. Obers, in: "Infinite Analysis"; Int. J. Mod. Phys. A7, [Suppl. 1A] (1992) 339.

[20] I. Bars and C. Kounnas, Phys. Rev. D56 (1997) 3664. 